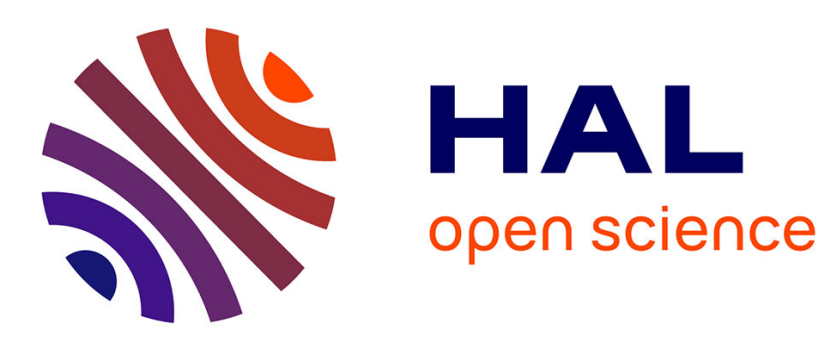

\title{
Le workfare ou la mise au travail gratuit des allocataires de l'aide sociale
}

\author{
Maud Simonet
}

\section{To cite this version:}

Maud Simonet. Le workfare ou la mise au travail gratuit des allocataires de l'aide sociale. Regards croisés sur l'économie, 2021, Rémunérer le travail, 27, pp.184-193. 10.3917/rce.027.0184 hal03454511

\section{HAL Id: hal-03454511 https://hal.science/hal-03454511}

Submitted on 6 Jan 2022

HAL is a multi-disciplinary open access archive for the deposit and dissemination of scientific research documents, whether they are published or not. The documents may come from teaching and research institutions in France or abroad, or from public or private research centers.
L'archive ouverte pluridisciplinaire HAL, est destinée au dépôt et à la diffusion de documents scientifiques de niveau recherche, publiés ou non, émanant des établissements d'enseignement et de recherche français ou étrangers, des laboratoires publics ou privés. 


\title{
Le workfare ou la mise au travail gratuit des allocataires de l'aide sociale
}

\author{
Maud Simonet (CNRS, IDHES UMR8533)
}

Dans Regards croisés sur l'économie 2020/2 (n²7), pages 184 à 193

Créé à partir de la contraction du terme welfare - aide sociale — et du terme work

— travail —, le workfare, entreprise conservatrice qui oblige les allocataires de l'aide sociale à travailler pour percevoir leur allocation, s'est institutionnalisé aux États-Unis dans les dernières décennies du $\mathrm{xx}^{\mathrm{e}}$ siècle. Après avoir présenté les étapes et les fondements moraux, genrés et racistes de cette réforme de l'assistance, cet article montre comment

l'institutionnalisation de ce travail gratuit et contraint vient brouiller les frontières du salariat et participe d'une nouvelle régulation du marché du travail, qui rend ce dernier invisible et gratuit.

\section{Le «workfare » : jalons d'un néologisme, récit d'une expansion}

$\underline{2}$ On retient souvent comme date de naissance officielle du workfare l'année 1996 avec l'adoption, aux États-Unis, de la Personal Responsibility and Work Opportunity

Reconciliation Act (PRWORA) qui impose à tous les États d'instaurer une obligation de travail pour les allocataires de l'aide sociale. Mais cette réforme qui promet d'« en finir avec l'aide sociale telle que nous la connaissons » pour reprendre la célèbre formule de Bill Clinton, a des racines plus anciennes. Comme le montre bien Sylvie Morel (2000), la mise au travail comme forme étatique de régulation de la pauvreté jalonne l'histoire de l'Amérique, depuis les workhouses héritées de l'ancienne puissance coloniale au Civil Work Administration qui fait travailler les chômeurs pendant le New Deal. Mais l'introduction du workfare à proprement parler remonte aux années 1970, sous l'administration Nixon, au cours de laquelle plusieurs projets pilotes obligeant les allocataires à travailler en compensation de leurs allocations sont expérimentés dans différents États. C'est d'ailleurs à Nixon que l'on attribue la première occurrence du terme « workfare», lors d'un discours télévisé en août 1969 : «What America needs now is not more welfare but more "workfare" [1][1]« Ce dont l'Amérique a besoin maintenant, ce n'est pas de plus.... » (Peck, 2001, p. 90) C'est dans la décennie suivante que le workfare s'impose véritablement comme un thème clé de l'agenda politique conservateur. Reagan, qui fera vigoureusement campagne sur la lutte contre l'assistanat et contre la fraude à l'aide sociale, met en place, dès son arrivée au pouvoir, plusieurs mesures budgétaires (Omnibus Budget Reconciliation Act, 1981) puis législatives (Family Support Act, 1988) visant à la fois à restreindre l'aide sociale fédérale et à conditionner son versement à l'exécution d'un travail pour de nombreuses catégories de bénéficiaires ; parmi elles, les mères avec des enfants en bas âge, principale catégorie soutenue par l'aide sociale à travers l'AFDC (Aid to Family with Dependant Children) créée en 1935.

3 Dans les années 1990, la question de la réforme de l'aide sociale devient omniprésente dans le débat public américain, bien au-delà des conservateurs. La loi signée par Clinton en 1996 et 
soutenue par les députés du Parti Républicain, vient en quelque sorte boucler cette séquence en institutionnalisant au niveau fédéral ce nouveau paradigme. Elle limite à cinq ans, pour chaque famille, l'aide du gouvernement fédéral et introduit une pénalisation des États qui ne parviennent pas à placer un certain nombre des bénéficiaires dans des activités de travail. Elle transforme également les modalités de financement de cette aide, incitant les États à baisser le nombre de bénéficiaires pour conserver une partie du reliquat des financements non utilisés (Krinsky, 2009). En une vingtaine d'années, le système d'aide sociale américain s'est largement transformé : non seulement le nombre de bénéficiaires de l'aide sociale a diminué mais leur mise au travail obligatoire a fortement modifié leur statut, sans nécessairement transformer leur identité et leur destinée sociales. Au cours de cette période, la signification du terme workfare s'est elle-même modifiée, devenant à la fois plus large et plus abstraite. Le terme ne recouvre plus uniquement des programmes qui obligent à compenser strictement le montant de l'allocation par un nombre d'heures de travail non rémunérées (work for welfare), mais évoque plus largement un système dont la logique, la structure et les dispositifs visent à « maximiser la participation par le travail en diminuant la "dépendance" à l'aide sociale » (welfare to work) (Peck, 2001). Comme le souligne l'économiste Bob Jessop au début du $\mathrm{xxi}^{\mathrm{e}}$ siècle, le workfare ne désigne plus les seuls « projets de prédilection des néoconservateurs et néo-libéraux » mais plus largement " une attaque bipartisane largement soutenue électoralement contre les États-providence des grandes économies capitalistes » (Jessop, 2003). Loin de se cantonner aux États-Unis, cette nouvelle norme de la mise au travail s'est en effet progressivement diffusée, d'abord au Royaume-Uni puis dans d'autre pays comme l'Australie, l'Allemagne, la Suisse, etc.

\section{Travailler gratuitement pour « racheter » sa citoyenneté}

4Le nouveau paradigme de l'aide sociale qui s'ouvre avec l'institutionnalisation du workfare aux États-Unis s'inscrit dans une rhétorique qui fait porter aux allocataires la responsabilité de leur situation, insiste sur les « obligations sociales de la citoyenneté » (Morel, 2000, p. 180) et le devoir qui serait le leur de rembourser leur dette à la société. "Pour chaque allocation il y a une contrepartie, pour chaque droit il y a un devoir », déclarait ainsi en 1998 l'ancien maire de New York, Rudolf Giuliani, à propos des programmes de workfare en vigueur depuis presque vingt ans dans la ville. Ce langage du « contrat social» et de la « réciprocité » comme principe moral de justification de la mise au travail explique sans doute en partie le succès idéologique du « projet workfariste », tant il résonne avec l'éthique civique du travail, « valeur fondamentale » de la société américaine. Comme l'ont bien montré les travaux de Judith Shklar, le travail est depuis la période jacksonienne un marqueur clé de la citoyenneté américaine qui s'est élaborée contre la figure de l'esclave et autour de celle du « citoyen travailleur », alors incarnée par le «white male craft worker » (Shklar, 1991).

5Cette définition genrée et racisée de la «bonne » citoyenneté va se retrouver au cœur de la critique conservatrice du welfare, comme en témoigne la violente attaque contre la «welfare queen » autour de laquelle Reagan fera campagne en 1976. Dépeinte sous les traits d'une « femme noire des banlieues de Chicago se jouant de l'État social américain en cultivant le goût de la paresse » (Périvier, 2012), cette figure de la « welfare mother », non fondée statistiquement mais forte d'une charge symbolique liée aux différents rapports sociaux qu'elle condense, va bientôt incarner dans le débat politique lancé par les promoteurs du workfare la « quintessence de la dépendance » (Fraser et Gordon, 1994), la figure repoussoir 
de la « non citoyenneté ». En poussant jusqu'aux mères célibataires sur le marché du travail, le workfare impose donc aux allocataires de l'aide sociale de faire la preuve, par leur travail gratuit, de leur appartenance à la communauté des « citoyens travailleurs » et de « se mettre en conformité à un modèle de comportement dominé par l'éthique du travail » (Morel, 2000, p. 188). Niant le travail domestique et familial que ces femmes à l'aide sociale fournissent pourtant au quotidien et leur refusant le « soutien financer dont elles ont besoin pour élever leur enfant à plein temps », ce nouveau paradigme de l'aide sociale est bien loin pour autant de les reconnaître « comme des travailleuses salariées à part entière » (Roberts, 2004).

\section{Brouillage des frontières du salariat et « gratuitisation » du travail}

6Dans son analyse du développement et de la contestation du workfare dans la ville de New York dans les années 1980-1990, John Krinsky souligne combien ces workfare workers, qui travaillaient parfois entre vingt et quarante heures par semaine en échange de leurs allocations, n'avaient pas accès à la représentation syndicale, à la protection légale contre le harcèlement sexuel, ou tout simplement à des conditions de rémunération et de travail décentes. Essentiellement placé·es dans les services municipaux, ils et elles étaient plus de 6000 au cours des années 1990 à nettoyer les rues, les parcs ou les stations du métro de New York, à côté et, de plus en plus, à la place de fonctionnaires municipaux dont le nombre, lui, diminuait drastiquement depuis la crise budgétaire des années 1970 (Krinsky, 2007). Partageant leur quotidien et une partie de leurs tâches, ils et elles n'en partageaient ni le salaire, ni les droits, ni les possibilités de carrière. Mieux, ce développement d'une maind'œuvre invisible et précaire, en dehors du pouvoir syndical et du droit salarial, chargée de racheter, par ses heures de travail, sa citoyenneté, a constitué « une stratégie parmi d'autres de flexibilisation de la main-d'œuvre publique » (Krinsky, 2009). À la suite de nombreuses mobilisations dénonçant ce programme et ses usages par la municipalité au début des années 2000, celle-ci crée la place un « programme transitionnel vers l'emploi » (le Job Training Program) dans lequel les allocataires seront un peu payé·es, mais toujours bien moins que le premier échelon de la fonction municipale au travail à leur côté. S'ils et elles parviennent à obtenir quelques droits « salariaux » et syndicaux au fil du temps, ceux-ci restent toutefois incomplets.

7" Workfare is not about creating jobs for people that don't have them, it is about creating workers for jobs that nobody wants [2][2] « Le workfare ne vise pas à créer des emplois pour des... " écrivait Jamie Peck dans Workfare States en 2001. Il invitait ainsi à renverser la présentation habituelle de cette réforme — remettre les assisté·es au travail — et à prendre au sérieux sa participation à une nouvelle régulation du marché du travail par la construction d'une « nouvelle catégorie de travailleur'ses forcé·es » (Peck, 2001, p. 188) contraint·es d'accepter du travail pas, ou très mal, payé, « au nom de la citoyenneté » (Simonet, 2018). Comme en atteste l'exemple de New York, la variété des programmes de workfare ou de welfare to work a permis la construction progressive d'une zone grise entre le workfare pur et l'emploi municipal qui contribue aujourd'hui à brouiller et surtout à élargir la frontière entre le travail gratuit, et invisible comme tel, et le salariat (Krinsky et Simonet, 2012). Au cours de la même période, dans la ville de New York, une autre catégorie de travailleur'ses invisibles et gratuit·es est apparue à côté des allocataires de l'aide sociale et des fonctionnaires municipaux dans les parcs. Il s'agit des bénévoles, à majorité des femmes, issues des classes moyennes et supérieures, massivement sollicitées par la municipalité et des partenariats 
publics-privés pour s'engager dans le nettoyage des parcs et jardins de la ville. Ces bonnes citoyennes, largement célébrées comme telles, ont en quelque sorte incarné l'autre face de la " gratuitisation » du travail dans ce petit service public municipal, s'attaquant par un autre angle - celui du travail gratuit choisi et valorisé et non du travail gratuit contraint et stigmatisant — aux frontières du salariat, public, protégé, syndiqué et largement masculin en charge de l'entretien des parcs.

\section{Conclusion : la contrepartie en «bénévolat », un workfare à la française?}

$\underline{8}$ Il y a exactement vingt ans, Jamie Peck écrivait : « alors que le workfare a acquis un statut de mantra politique aux États-Unis, le mot est resté jusqu'à peu tabou dans les démocraties sociales européennes. » (2001, p. 85) Si l'expansion considérable du workfare en Angleterre depuis les années Blair ou le développement « des occasions de travail indemnisées », plus connues sous le nom de « jobs à 1 euro », en Allemagne, y ont depuis rendu caduque ce tabou, les travaux des spécialistes de la protection sociale ont longtemps souligné que la France ne s'inscrivait pas dans le paradigme du « workfare » mais dans celui de «l'insertion », le travail y étant vu « comme facteur de promotion vers plus d'intégration citoyenne, et non comme forme de punition ou de "contrepartie" » (Barbier, 2008). La question de la « contrepartie » au RSA revient pourtant régulièrement, depuis quelques années, dans le débat politique national, depuis la proposition de Laurent Wauquiez en 2011 d'instaurer cinq heures de travail d'utilité sociale pour les allocataires du RSA, aux déclarations d'Édouard Philippe en 2019 sur « la vraie belle question politique » que serait « la contrepartie ». Mieux, depuis l'arrêt du Conseil d'État du 15 juin 2018, il est désormais possible pour un département de prévoir, dans le contrat conclu avec une personne bénéficiaire du RSA, des actions de «bénévolat » et de nombreuses municipalités ou départements ont déjà mis en place de tels programmes, plus ou moins « volontaires » pour « leurs » allocataires (Simonet, 2018). Ils et elles sont alors invité es à faire la preuve de leur « utilité sociale » et ce faisant de leur appartenance à la communauté des « citoyen·nes travailleur'ses » en nettoyant gratuitement, en échange de leurs allocations, les parcs, les rues et les plages des collectivités.

\section{Notes}

- [1]

« Ce dont l'Amérique a besoin maintenant, ce n'est pas de plus d'aide sociale mais de plus de "workfare". »

- [2]

« Le workfare ne vise pas à créer des emplois pour des personnes qui n'en ont pas, mais à créer des travailleurs pour des emplois dont personne ne veut. » 